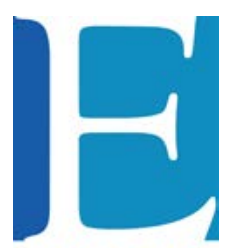

EVALUAR
2017, Vol. 17, No. 1

ISSN $1667-4545$

Recuperado de www.revistas.unc.edu.ar

Laboratorio de Evaluación Psicológica y Educativa Facultad de Psicología - Universidad Nacional de Córdoba

\title{
Conflictos entre Pares en el Aula y Estilos de Manejo de Conflictos en Estudiantes de Bachillerato
}

\section{Conflicts with Classmates and Conflict Management Styles in High School Students}

\author{
Alejandro César Antonio Luna-Bernal * 1, Juan Carlos Mejía-Ceballos ${ }^{2}$, \\ Francisco Augusto Laca-Arocena ${ }^{3}$ \\ 1. Universidad de Guadalajara, México. \\ 2. Universidad Autónoma del Estado de Morelos, México. \\ Introducción \\ Método \\ Resultados \\ Discusión \\ Referencias
}

3. Universidad de Colima, México.

Recibido: 07/02/2017 Revisado: 16/02/2017 Aceptado: 03/03/2017

\section{Resumen}

El presente trabajo se planteó tres objetivos: a) identificar el grado de frecuencia e intensidad de conflictos que los adolescentes estudiantes de bachillerato perciben tener con sus compañeros de aula en relación a determinados temas de conflicto; b) analizar posibles diferencias de género, edad y grado escolar en la frecuencia e intensidad percibidas, y c) examinar la posible relación de la frecuencia e intensidad con los estilos de manejo de conflictos que los adolescentes afirman emplear en las interacciones con sus compañeros. Participaron 171 adolescentes estudiantes de bachillerato de una escuela pública ubicada en Guadalajara, México, con edades de 15 a 19 años, quienes respondieron el Cuestionario sobre Conflictos con Compañeros de Aula. En los resultados se encontraron diferencias significativas por género, edad y grado escolar, así como correlaciones directas entre frecuencia e intensidad de conflictos y estilos de manejo de conflictos. Se discuten estos y otros resultados en el marco de la literatura sobre gestión de conflictos en la adolescencia.

Palabras clave: adolescencia, conflicto, resolución de conflictos

\begin{abstract}
This study addressed three objectives: a) to identify the degree of frequency and intensity of conflicts that high school adolescents perceive to have with their classmates regarding certain conflict issues; b) to analyse possible differences in perceived frequency and intensity according to gender, age and grade; and c) to examine possible relationships between frequency and intensity of conflicts, and conflict management styles. The test subjects were 171 students from a public high school in Guadalajara (México) aged 15 to 19 years, who answered the Questionnaire on Conflicts with Classmates. Significant differences were found related to gender, age and grade level, as well as direct correlations between frequency and intensity of conflicts, and conflict management styles. These and other findings are discussed considering previous literature on conflict management in adolescence.
\end{abstract}

Keywords: adolescence, conflict, conflict resolution 


\section{Introducción}

Los conflictos pueden definirse como situaciones en las cuales dos o más sujetos perciben sus respectivos intereses como incompatibles (Rubin, Pruitt, \& Kim, 1994). En la actualidad, diversos autores han llamado la atención sobre el hecho de que los conflictos, así entendidos, son inherentes a la condición humana y no implican necesariamente agresión, ya que los seres humanos hemos creado distintos mecanismos para manejar esas situaciones de modos no violentos (p. ej., Fry, 2006; Galtung, 2003; Martínez-Guzmán, 2009; Paris-A1bert, 2009). En ese sentido, diversos autores han señalado la importancia de distinguir, en la investigación con adolescentes, el fenómeno del conflicto del de la violencia (p. ej., Bascón, Saavedra, \& Arias, 2013; Pantoja-Vallejo, 2005).

Sin embargo, a pesar de la importancia que reviste el estudio del rol que cumplen los conflictos cotidianos en la vida diaria de los adolescentes, este aspecto de la vida escolar ha sido poco abordado. El énfasis de la literatura en el área ha tendido a centrarse en el estudio de los fenómenos de violencia escolar (especialmente, el bullying). En ese marco, actualmente es posible constatar la escasez de estudios que proporcionen datos sobre los principales temas de conflicto cotidianos entre pares adolescentes en el aula, analizando sus características de frecuencia e intensidad. Esto ocurre pese al interés creciente que existe en diversos ámbitos por el análisis del conflicto y sus formas de resolución.

Por lo anterior, y con el fin de contribuir a llenar ese vacío, el objetivo de este estudio es realizar una aproximación a esta problemática en nuestro contexto mediante un análisis de, por una parte, los conflictos que se presentan entre compañeros de aula y, por otra, su relación con los estilos de manejo de conflictos manifestados por los estudiantes.

En concreto, se plantearon tres objetivos particulares para el presente trabajo: a) identifi- car el grado de frecuencia e intensidad de conflictos que los adolescentes estudiantes de bachillerato perciben tener con sus compañeros de aula en relación a determinados temas de conflicto; b) analizar posibles diferencias de género, edad y grado escolar en cuanto a frecuencia e intensidad percibidas, y c) examinar la posible relación entre la frecuencia e intensidad de los conflictos y los estilos de manejo de conflictos que los adolescentes manifiestan emplear en las interacciones con sus compañeros.

\section{Conflictos entre compañeros en el aula}

De acuerdo con Laursen y Collins (1994), la expresión temas de conflicto (conflict issues) hace referencia a los asuntos respecto de los cuales se producen los conflictos. En el caso de los conflictos en el aula, algunos temas de conflicto entre pares se dan por causas como hacer ruido en el salón, los chismes, las calificaciones, las tareas o las burlas. La frecuencia de conflictos ha sido definida en diversos estudios como la cantidad de veces que las personas tienen conflictos respecto a un determinado tema. Asimismo, la intensidad se ha definido como el nivel de tensión emocional percibido por los involucrados durante tales discusiones (Laursen \& Koplas, 1995; Luna-Bernal \& Cruz-Abundis, 2014; Luna-Bernal, Laca-Arocena, \& Cruz-Abundis, 2013; Parra-Jiménez \& Oliva-Delgado, 2002; Pérez-Ramos \& Pineda-Sánchez, 2013).

Como se señaló, son escasos los estudios sobre temas de conflicto cotidianos entre adolescentes en el contexto escolar. En la revisión bibliográfica llevada a cabo para el presente trabajo se identificaron los estudios llevados a cabo por Delpino-Goicochea (2011) y por Eresta-Plasín y Delpino-Goicochea (2009) en España, y el estudio de Castro-Guerrero, López-Jiménez y Meza-Altamar (2011) desarrollado en Colombia, además de un par de estudios efectuados con estudiantes mexicanos de bachille- 
rato (Luna-Bernal \& Laca-Arocena, 2011) y de secundaria (Luna-Bernal, De Gante-Casas, \& Gómez-Pérez, 2015). A continuación, resumiremos algunos de los principales resultados de estos estudios.

El estudio de Castro-Guerrero et al. (2011) fue realizado con 60 estudiantes de noveno grado de una institución pública del municipio de Malambo, Atlántico, Colombia. Se les preguntó a los participantes sobre las causas que con mayor relevancia generan conflictos en la escuela. Los estudiantes identificaron como situaciones de conflicto el enfrentamiento entre los alumnos y el profesor, las malas palabras en clase, que no se respetan las normas, que los alumnos se insultan, que hay peleas, y que hay pequeños grupos que no se llevan bien. También los estudiantes consideraron como temas de conflicto el no traer el uniforme, las llegadas tarde y el escaparse de clase. La mayoría de los encuestados consideraron que las malas palabras son las que generan más acciones conflictivas, seguidas por las llegadas tarde. De acuerdo con los autores, a pesar de que reconocen la existencia de estas dificultades de convivencia, en general, los adolescentes opinaron que sus relaciones pueden considerarse normales.

Por su parte, en España, Delpino-Goicochea (2011) llevó a cabo un estudio con una muestra de 1,412 adolescentes con edades entre 15 y 18 años. Los participantes estaban matriculados en 25 centros educativos tanto públicos como privados, ubicados en siete ciudades españolas. Además, se realizaron cuatro grupos de discusión con 39 estudiantes. La autora llamó la atención sobre el hecho de que los adolescentes tendieron a referirse a los conflictos como "problemas". Con relación a la pregunta acerca de aquellos comportamientos considerados problema en las relaciones entre pares en el aula, se ubicaron en primer lugar las burlas (29.4\%), seguidas de las conductas molestas en clase (16.1\%), las agresiones físicas (16\%), el ignorar a otros $(9 \%)$ y el rechazo a quienes son de otro país $(6.3 \%)$.

En México, encontramos como primer antecedente un estudio llevado a cabo por Luna-Bernal y Laca-Arocena (2011) con 282 adolescentes estudiantes de bachillerato de las ciudades de Guadalajara y Colima, con un rango de edad de 14 a 19 años. En ese estudio, los autores desarrollaron un Cuestionario sobre Conflictos con Compañeros de Aula, mediante el cual evaluaron la frecuencia e intensidad de los conflictos entre adolescentes estudiantes de bachillerato con respecto a 21 temas de conflicto, considerando diferencias de género y edad. En los resultados se verificó que los participantes tendían a presentar mayores conflictos con compañeros del mismo género, y se manifestó una mayor inclinación de las mujeres hacia los conflictos relacionales.

Un segundo antecedente en México para nuestro trabajo es el estudio llevado a cabo por Luna-Bernal et al. (2015), con una muestra ( $\mathrm{N}=$ 404) de estudiantes de secundaria pertenecientes a dos planteles públicos de la Zona Metropolitana de Guadalajara, Jalisco. El rango de edad de los participantes fue de 11 a 15 años. Los autores emplearon el Cuestionario sobre Conflictos con Compañeros de Aula elaborado previamente por Luna-Bernal y Laca-Arocena (2011). Los temas de conflicto más frecuentes e intensos entre los adolescentes resultaron ser los relativos a faltas de respeto o insultos, creerse más que los demás, chismes y burlas. Por su parte, los temas de conflicto menos frecuentes fueron los relativos al dinero y a copiar o pasar las respuestas en los exámenes. En cuanto a las diferencias de género, las mujeres obtuvieron puntajes más altos que los hombres en los conflictos por chismes y en los conflictos por hipocresía o envidia, tanto en frecuencia como en intensidad de conflictos. Por su parte, los hombres presentaron mayor frecuencia que ellas en los conflictos relativos a agarrar las cosas de los demás y resultaron tener conflictos más intensos que las mujeres en el tema relativo a la di- 
visión de tareas en equipo. Los autores también encontraron, tanto en la frecuencia como en la intensidad de los conflictos, una tendencia a aumentar según el grado escolar y la edad de los estudiantes participantes.

Como se puede observar, los antecedentes de estudios sobre temas de conflictos cotidianos entre adolescentes en la escuela hasta el momento, parecen mostrar que los adolescentes tienden a percibir una frecuencia e intensidad entre baja y moderada en la incidencia de tales conflictos y que se inclinan a verlos como problemas de relación que son normales en sus interacciones. No obstante, como se puede apreciar, es probable que existan diferencias de género, grado y edad que es importante considerar a fin de comprender de manera más precisa la dinámica y el significado de estos conflictos cotidianos para el desarrollo psicosocial de los adolescentes, así como su relación con los modos de conducta que utilizan para afrontarlos.

\section{Estilos de manejo de conflictos}

De acuerdo con Filley (1975/1985), los estilos personales de manejo de conflictos pueden definirse como "los patrones de comportamiento que las personas utilizan para resolver conflictos" (p. 60). La presente investigación se basará en el modelo de Ross y DeWine (1988) debido a que este modelo se ha mostrado consistente y útil para estudiar los estilos de manejo de conflictos en adolescentes (p. ej., Luna \& Laca, 2014; Luna-Bernal, 2014; Luna-Bernal \& De Gante-Casas, 2017). Ross y DeWine (1988) propusieron tres estilos comunicativos para el manejo de conflictos: a) Centrado en Uno Mismo, b) Centrado en la Otra Parte y c) Centrado en el Problema.

El estilo Centrado en Uno Mismo se presenta cuando en el manejo de un conflicto el individuo asume una actitud competitiva y refleja en sus mensajes un énfasis en los intereses pro- pios. Por ejemplo, en mensajes como "¡Cállate, estás equivocado(a)! No quiero oír nada más de lo que tengas que decir", "Es tu culpa si yo fallo en esto, y jamás esperes alguna ayuda de mi parte cuando estés en un apuro", o "¡No puedes hacerme (o decirme) eso; de todos modos es a mi manera. ¡Olvídalo!”, el sujeto enfatiza su posición personal incluso a costa de sacrificar o de no considerar el interés de la contraparte.

El estilo Centrado en la Otra Parte, por el contrario, se presenta cuando el sujeto asume actitudes de acomodación emitiendo mensajes con los que manifiesta su voluntad de pasar por alto el problema y de complacer a la contraparte. Por ejemplo, en mensajes como "¿Cómo puedo hacerte sentir bien otra vez?”, "Cualquier cosa que te haga sentir mucho mejor está bien para mí,, o "Lamento mucho que te sientas herido(a); quizás tú tengas razón", el sujeto busca satisfacer el interés de la otra parte, incluso mostrándole completa disposición o dándole la razón.

Por último, el estilo Centrado en el Problema se presenta cuando en el manejo de conflictos el sujeto emite mensajes en los que asume una actitud de colaboración o compromiso, considerando que el conflicto se puede resolver atendiendo a los intereses de ambas partes sin arriesgar la relación. Por ejemplo, en mensajes como "Estoy muy molesto por algunas cosas que están pasando, ¿podemos hablar sobre ellas?”, “Qué posibles soluciones podemos encontrar?", o "Intentemos encontrar una solución que nos dé a cada uno algo de lo que queremos", el sujeto solicita hablar sobre el problema, pregunta sobre las posibles soluciones e invita a la contraparte a encontrar una solución negociada.

\section{Método \\ Participantes}

La muestra del presente estudio $(\mathrm{N}=171)$ estuvo compuesta por estudiantes de un bachi- 
1lerato público ubicado en la Zona Metropolitana de Guadalajara, Jalisco (México), de los cuales $91(53.2 \%)$ eran mujeres y $80(46.8 \%)$ varones. El rango de edad fue de 15 a 19 años $(\mathrm{M}=16.70 ; \mathrm{DE}=.94)$. Los participantes se encontraban distribuidos en los seis grados (semestres) que comprende el bachillerato general en México.

Para analizar la variable edad en el presente estudio se formaron dos grupos: de $15 \mathrm{y}$ 16 años y de 17 a 19 años. Con relación a la variable de grado escolar, se decidió analizarla por anualidades, considerando tres niveles: Primer año (semestres 1 y 2), Segundo año (semestres 3 y 4), y Tercer año (semestres 5 y 6). En la Tabla 1 se puede observar la distribución por género según la edad y el grado escolar. frecuencia se le pide al participante responder si con relación a cada uno de los temas mencionados se presentan entre él y sus compañeros: 1 (Ningún conflicto), 2 (Algunos conflictos), 3 (Bastantes conflictos), ó 4 (Muchos conflictos). En la escala de intensidad se le pide al adolescente responder si se trata de: 1 (Ningún conflicto), 2 (Conflictos leves), 3 (Conflictos de intensidad media), ó 4 (Conflictos fuertes). Para su calificación, se calcula la media aritmética de los 23 reactivos para cada una de las dos escalas, obteniéndose así un puntaje de frecuencia y otro de intensidad de conflictos. Además de este puntaje global de cada una de las escalas, en el presente trabajo se analizaron por separado cada uno de los 23 temas de conflicto.

\section{Tabla 1}

Distribución de la muestra por género, edad y grado escolar.

\begin{tabular}{llll}
\hline & Mujeres & Hombres & Total \\
\hline 15 y 16 años & $36(21.1 \%)$ & $35(20.5 \%)$ & $71(41.5 \%)$ \\
17 a 19 años & $55(32.2 \%)$ & $45(26.3 \%)$ & $100(58.5 \%)$ \\
Primer año & $33(19.3 \%)$ & $29(17.0 \%)$ & $62(36.3 \%)$ \\
Segundo año & $28(16.4 \%)$ & $30(17.5 \%)$ & $58(33.9 \%)$ \\
Tercer año & $30(17.5 \%)$ & $21(12.3 \%)$ & $51(29.8 \%)$ \\
Total & $91(53.2 \%)$ & $80(46.8 \%)$ & $171(100 \%)$ \\
\hline
\end{tabular}

Nota. Los porcentajes son en relación con la muestra total.

\section{Instrumentos}

\section{Cuestionario sobre Conflictos con Compañe-} ros de Aula. Este instrumento informa sobre la frecuencia e intensidad de conflictos que los participantes perciben tener con sus compañeros de aula, con relación a 23 temas de conflicto. El instrumento se compone de 23 reactivos, cada uno de los cuales hace referencia a un posible tema de conflicto entre compañeros. Por ejemplo, Por dinero (reactivo 1), Por chismes (reactivo 3), Por burlas (reactivo 4). Respecto de cada tema, el participante debe señalar la frecuencia con que ha experimentado dichos conflictos así como su intensidad. En la escala de
Cabe señalar que, al final de la lista de los 23 reactivos, también se presenta al participante un espacio para que pueda agregar libremente hasta tres reactivos (temas) nuevos. Para ello, se le da la indicación de que anote él mismo otros temas sobre los que haya tenido conflictos con sus compañeros y que no estén ya incluidos en la lista. En caso de que los participantes añadan nuevos temas en esta sección del instrumento, no se computarán para el presente estudio sino que solamente se tomarán en cuenta para ser agregados en futuras versiones del Cuestionario.

Como se señaló anteriormente, el Cuestionario sobre Conflictos con Compañeros de 
Aula fue desarrollado originalmente por Luna-Bernal y Laca-Arocena (2011), en base al diseño de la Escala de Conflicto Familiar en la Adolescencia (ECFA) de Parra-Jiménez y Oliva-Delgado (2002). Como se explicó más arriba, el instrumento original de Luna-Bernal y Laca-Arocena (2011) quedó conformado por 21 reactivos.

Con respecto al presente trabajo conviene señalar dos puntos. En primer lugar, por sugerencia de algunos adolescentes participantes en los estudios anteriores, así como por consideraciones terminológicas y conceptuales, se tomó la decisión de separar los conceptos de hipocresía y envidia y, por lo tanto, se modificó el reactivo número 9 que en la versión original decía "Por hipocresía o envidia". En la presente versión el reactivo 9 se reemplazó por Por hipocresía y se añadió el reactivo 22: Por envidia. También por sugerencia de algunos participantes, se añadió el nuevo reactivo 23: Por ser eliminado o no aceptado en Facebook. De esta manera, la versión del instrumento que será utilizada en el presente estudio quedó conformada por 23 reactivos.

En segundo lugar, a fin de obtener información sobre la validez y confiabilidad de las escalas de frecuencia e intensidad de conflictos considerando los datos del presente estudio, se realizó un análisis de componentes principales de ambas escalas, así como también un análisis de confiabilidad alfa de Cronbach de las mismas, tal como se señala enseguida.

En cuanto a la escala de Frecuencia de Conflictos se obtuvieron valores adecuados en la medida Kaiser-Mayer-Olkin y en la prueba de esfericidad de Bartlett $\left(\mathrm{KMO}=.799 ; \chi^{2}{ }_{[253]}\right.$ $=1080.622, p<.01)$, lo cual indicó la pertinencia del análisis de componentes principales para los datos muestrales. En dicho análisis se obtuvo una solución monofactorial que explica el $25.46 \%$ de la varianza con un índice de confiabilidad alfa de Cronbach de .86 para el total de la escala. El Factor I quedó conformado por los siguientes 23 reactivos (carga factorial entre paréntesis, en orden descendente): 19 (.602), 10 (.591), 3 (.585), 16 (.579), 22 (.571), 4 (.571), 9 (.548), 11 (.544), 14 (.540), 20 (.538), 6 (.537), 18 (.536), 8 (.521), 5 (.486), 7 (.480), 13 (.478), 15 (.460), 21 (.450), 12 (.409), 17 (.404), 23 (.388), 2 (.374) y 1 (.237).

En cuanto a la escala de Intensidad de Conflictos se obtuvieron valores adecuados en la medida Kaiser-Mayer-Olkin y en la prueba de esfericidad de Bartlett $\left(\mathrm{KMO}=.816 ; \chi^{2}{ }_{[253]}\right.$ $=1148.271, p<.01$ ), lo cual indicó la pertinencia del análisis de componentes principales para los datos muestrales. En dicho análisis de componentes se obtuvo una solución monofactorial que explica el $26.63 \%$ de la varianza con un índice de confiabilidad alfa de Cronbach de .872 para el total de la escala. El Factor I quedó conformado por los siguientes 23 reactivos (carga factorial entre paréntesis, en orden descendente): 5 (.622), 4 (.615), 6 (.606), 10 (.584), 8 (.562), 3 (.557), 16 (.551), 12 (.551), 7 (.549), 18 (.546), $14(.545), 19(.530), 11(.523), 17$ (.520), 20 (.504), 9 (.501), 2 (.493), 22 (.485), 13 (.465), 21 (.394), 15 (.372), 1 (.319) у 23 (.319).

\section{Cuestionario sobre Estilos de Mensajes en} el Manejo de Conflictos (Ross-DeWine Conflict Management Message Style instrument, CMMS). Este cuestionario informa sobre la frecuencia con que los participantes perciben utilizar tres estilos de mensajes en el manejo de conflictos según el modelo de Ross y DeWine (1988). Cada uno de los reactivos del CMMS es una oración que representa un mensaje dado por un individuo a su contraparte en una situación de conflicto interpersonal, por ejemplo: ¿Cómo puedo hacerte sentir bien otra vez? (reactivo 2) o Estoy muy molesto por algunas cosas que están pasando; ¿podemos hablar sobre ellas? (reactivo 3), o bien ;Cállate, estás equivocado(a)! No quiero oír nada más de lo que tengas que decir (reactivo 8). Para contestar, se le pide al 
participante que responda con qué frecuencia él ha utilizado, en sus conflictos, mensajes similares a los representados por cada reactivo. El formato de respuesta es una escala Likert de cinco puntos donde el 1 corresponde a Nunca digo cosas como esto, el 2 a Rara vez digo cosas como esto, el 3 a Algunas veces digo cosas como esto, el 4 a $A$ menudo digo cosas como esto y el 5 a Generalmente digo cosas como esto. Para la calificación del CMMS se obtienen las medias y desviaciones estándar de los participantes en cada una de las tres escalas.

El CMMS fue elaborado originalmente por Ross y DeWine (1988) trabajando con muestras de 585, 210 y 123 estudiantes universitarios con edades promedio de 20 años. Mejía-Ceballos y Laca-Arocena (2006) realizaron la traducción y primera validación del CMMS a la lengua castellana. Posteriormente, Laca, Mejía y Mayoral (2011) llevaron a cabo un análisis factorial confirmatorio con una muestra de estudiantes universitarios mexicanos y españoles $(\mathrm{N}$ $=441$ ) con una media de edad de 21 años. Finalmente, Luna y Laca (2014) llevaron a cabo un amplio estudio de validación con una muestra $(\mathrm{N}=1,074)$ de estudiantes mexicanos de secundaria, bachillerato y licenciatura con un rango de edad de 11 a 25 años. La finalidad de este nuevo estudio fue analizar la estructura factorial del instrumento en una muestra de adolescentes y jóvenes, ya que los estudios anteriores habían sido efectuados con estudiantes universitarios. Una vez realizados los análisis factoriales (exploratorio y confirmatorio) por Luna y Laca (2014), el instrumento quedó compuesto por 13 reactivos divididos en tres escalas: estilo Centrado en Uno Mismo $(\alpha=.68)$, estilo Centrado en la Otra Parte $(\alpha=.72)$ y estilo Centrado en el Problema $(\alpha=.83)$, con una varianza explicada total de $56.10 \%$.

\section{Procedimiento}

Habiendo obtenido el permiso y la colaboración correspondientes por parte de las autoridades escolares, se solicitó a los estudiantes su participación dentro del aula de clases, en el horario acordado con la Institución. Se explicó a los estudiantes el objetivo de la investigación y se les invitó a participar de manera absolutamente voluntaria y anónima. Se les explicó que en estos instrumentos no hay respuestas buenas ni malas y se les invitó a contestar con sinceridad. Se les garantizó el manejo estrictamente confidencial y estadístico de la información y su uso para fines exclusivamente científicos.

\section{Análisis estadístico}

Con relación al primer objetivo particular del presente trabajo, que consistía en identificar el grado de frecuencia e intensidad de conflictos percibido por los participantes, se calcularon las medias y desviaciones estándar de frecuencia e intensidad de conflictos, tanto de la puntuación global como por temas de conflicto.

Con relación al segundo objetivo, que consistía en analizar posibles diferencias de género, edad y grado escolar, se examinaron dichas diferencias tanto en los puntajes globales de frecuencia e intensidad, como en cada uno de los 23 temas de conflicto.

Cabe mencionar que al revisar los supuestos necesarios para la realización de las pruebas paramétricas, se encontró que en algunas de las variables correspondientes a los 23 temas de conflicto no se alcanzaba a cubrir el supuesto de normalidad. Es por esto que se decidió emplear pruebas no paramétricas en tales casos a fin de identificar si la diferencia podría ser estadísticamente significativa (Coolican, 2004/2005; Ritchey, 2008).

Así, tanto en el caso del análisis de las diferencias de género como en el de diferen- 
cias de edad, se empleó la prueba $t$ de Student (paramétrica) o la prueba $U$ de Mann-Whitney (no paramétrica), según si la variable a analizar cumplía o no con el supuesto de normalidad.

Por su parte, para analizar las diferencias por grado escolar se empleó la prueba ANOVA de un factor (paramétrica) o la de Kruskal-Wallis (no paramétrica). Para el análisis post hoc, se utilizó en el primer caso la Prueba Honestamente Significativa (HSD) de Tukey, y en el segundo caso el test de comparaciones múltiples de Dunn con corrección Bonferroni. En el apartado de Resultados, se reportan únicamente los casos en que las diferencias resultaron ser estadísticamente significativas a nivel $p<.05 \mathrm{o}$ $p<.01$.

Por último, respecto al tercer objetivo del presente trabajo, que consistía en analizar las relaciones entre los puntajes totales de frecuencia e intensidad de conflictos y los estilos de manejo de conflictos, se llevó a cabo un análisis correlacional de Pearson.
Todos los anteriores análisis fueron hechos empleando el programa estadístico SPSS versión 21.0 (IBM Corporation, 2012).

\section{Resultados \\ Estadística descriptiva}

Como se observa en la Tabla 2, la puntuación media total tanto en Frecuencia como en Intensidad de conflictos se ubicó en un lugar intermedio entre el punto 1 de la escala (Ningún conflicto) y el punto 2 (Algunos conflictos y Conflictos leves).

Asimismo, en la Tabla 2 puede observarse que los conflictos que obtuvieron las puntuaciones más elevadas tanto en Frecuencia como en Intensidad resultaron ser Por chismes, seguidos de Porque algunos se creen más que los demás, Por falta de respeto o insultos y Por burlas. Los conflictos menos frecuentes e intensos resultaron ser Por ser eliminado o no aceptado en Facebook y Por copiar o pasar respuestas en los exámenes.

\section{Tabla 2}

Medias y Desviaciones Estándar de Frecuencia e Intensidad de conflictos.

\begin{tabular}{llll}
\hline & & Frecuencia & Intensidad \\
\hline 1 & Por dinero & $1.39(.59)$ & $1.40(.65)$ \\
2 & Por los asientos o lugares en el salón & $1.33(.54)$ & $1.37(.62)$ \\
3 & Por chismes & $1.84(.84)$ & $1.91(.93)$ \\
4 & Por burlas & $1.74(.79)$ & $1.85(.91)$ \\
5 & Por favoritismos & $1.50(.75)$ & $1.53(.84)$ \\
6 & Por diferencias de opiniones o de manera de pensar & $1.71(.70)$ & $1.76(.82)$ \\
7 & Por falta de respeto o insultos & $1.75(.69)$ & $1.85(.82)$ \\
8 & Por la manera de hablar & $1.51(.71)$ & $1.50(.76)$ \\
9 & Por hipocresía & $1.68(.79)$ & $1.77(.93)$ \\
10 & Por la manera de vestir o arreglarse & $1.39(.68)$ & $1.45(.80)$ \\
11 & Por calificaciones & $1.50(.75)$ & $1.53(.84)$ \\
12 & Por hacer ruido o estar hablando en el salón & $1.73(.77)$ & $1.73(.85)$ \\
13 & Por agarrar las cosas de los demás & $1.39(.64)$ & $1.36(.67)$ \\
14 & Por la manera de ser de uno u otro & $1.39(.58)$ & $1.38(.62)$ \\
15 & Por el chico/a que nos gusta & $1.33(.61)$ & $1.36(.71)$ \\
16 & Porque algunos se creen más que los demás & $1.78(.80)$ & $1.84(.89)$ \\
17 & Por copiar o pasar las tareas de la escuela & $1.42(.62)$ & $1.40(.66)$
\end{tabular}




\begin{tabular}{llll}
\hline & & Frecuencia & Intensidad \\
\hline 18 & Por la división de tareas en equipo & $1.60(.66)$ & $1.63(.78)$ \\
19 & Por copiar o pasar respuestas en los exámenes & $1.22(.47)$ & $1.23(.57)$ \\
20 & Por su desempeño o mi desempeño durante los deportes o en competencias & $1.33(.66)$ & $1.39(.83)$ \\
21 & Por hacer lo que el otro quiere & $1.41(.62)$ & $1.43(.74)$ \\
22 & Por envidia & $1.62(.81)$ & $1.60(.84)$ \\
23 & Por ser eliminado o no aceptado en Facebook & $1.16(.46)$ & $1.19(.57)$ \\
Total & & $1.51(.34)$ & $1.54(.40)$ \\
\hline
\end{tabular}

Nota. $\mathrm{N}=171$.

\section{Diferencias de género}

Frecuencia de conflictos. La diferencia en el promedio total no fue estadísticamente significativa. Una vez hecho el análisis por temas de conflicto, se encontraron diferencias estadísticamente significativas sólo en tres temas de conflicto. En conflictos Por dinero $(U=2895.500$, $p<.01)$, los hombres $(\mathrm{M}=1.51 ; \mathrm{DE}=.64) \mathrm{ob}-$ tuvieron puntajes más altos que las mujeres $(\mathrm{M}$ $=1.27 ; \mathrm{DE}=.52)$. En conflictos Por hipocresía $\left(t_{[169]}=2.116, p<.05\right)$, las mujeres $(\mathrm{M}=1.80$; $\mathrm{DE}=.78)$ obtuvieron puntajes más altos que los hombres $(\mathrm{M}=1.55$; $\mathrm{DE}=.78)$. En conflictos Por la división de tareas en equipo $\left(t_{[169]}=\right.$ 2.056, $p<.05)$, las mujeres $(\mathrm{M}=1.69 ; \mathrm{DE}=$ .66) obtuvieron puntajes más altos que los hombres $(\mathrm{M}=1.49 ; \mathrm{DE}=.64)$.

Intensidad de conflictos. La diferencia en el promedio total no fue estadísticamente significativa. Una vez hecho el análisis por temas de conflicto, se encontraron diferencias estadísticamente significativas sólo en tres temas de conflicto. En conflictos Por dinero $(U=2902.500$, $p<.01)$, los hombres $(\mathrm{M}=1.54$; $\mathrm{DE}=.71)$ obtuvieron puntajes más altos que las mujeres $(\mathrm{M}$ $=1.27 ; \mathrm{DE}=.56)$. En conflictos Por hipocresía $(\mathrm{t}(169)=2.046, \mathrm{p}<.05)$, las mujeres $(\mathrm{M}=$ 1.90; $\mathrm{DE}=.92)$ obtuvieron puntajes más altos que los hombres $(\mathrm{M}=1.61 ; \mathrm{DE}=.92)$. En conflictos Por la división de tareas en equipo $\left(t_{[169]}\right.$ $=1.988, p<.05)$, las mujeres $(\mathrm{M}=1.74 ; \mathrm{DE}=$
.81) obtuvieron puntajes más altos que los hombres $(\mathrm{M}=1.50 ; \mathrm{DE}=.73)$.

\section{Diferencias por grupos de edad}

Frecuencia de conflictos. Pudo observarse una cierta tendencia del promedio general a aumentar con la edad siendo de $1.47(\mathrm{DE}=.34)$ para el grupo de 15-16 años, y de $1.54(\mathrm{DE}=.34)$ para el grupo de 17-19 años. Sin embargo, esta diferencia no resultó estadísticamente significativa.

En el análisis por temas de conflicto, se encontraron diferencias estadísticamente significativas sólo en dos temas de conflicto. En los conflictos Por hacer ruido o estar hablando en el salón $\left(t_{[169]}=2.025, p<.05\right)$ el grupo de 1719 años $(\mathrm{M}=1.83 ; \mathrm{DE}=.78)$ obtuvo puntajes más altos que el grupo de 15-16 años $(\mathrm{M}=1.59$; $\mathrm{DE}=.73)$. Por su parte, en los conflictos Por copiar o pasar respuestas en los exámenes $(U=$ $3053.000, p<.05)$ el grupo de 17-19 años $(\mathrm{M}=$ $1.26 ; \mathrm{DE}=.44)$ obtuvo puntajes más altos que el grupo de $15-16$ años $(\mathrm{M}=1.15 ; \mathrm{DE}=.50)$.

Intensidad de conflictos. También pudo observarse aquí una cierta tendencia del promedio general a aumentar con la edad, siendo de 1.49 $(\mathrm{DE}=.39)$ para el grupo de 15-16 años, y de $1.58(\mathrm{DE}=.40)$ para el grupo de 17-19 años. Sin embargo, esta diferencia no resultó estadísticamente significativa. 
En el análisis por temas de conflicto, se encontraron diferencias estadísticamente significativas sólo en dos temas de conflicto. En los conflictos Por hacer ruido o estar hablando en el salón $\left(t_{[169]}=2.208, p<.05\right)$, el grupo de 17 19 años $(\mathrm{M}=1.85 ; \mathrm{DE}=.86)$ obtuvo puntajes más altos que el grupo de 15-16 años $(\mathrm{M}=1.56$; $\mathrm{DE}=.81)$. En los conflictos Por la manera de ser de uno $u$ otro $(U=2946.500, p<.05)$, el grupo de 17-19 años $(\mathrm{M}=1.47$; $\mathrm{DE}=.67) \mathrm{ob}-$ tuvo puntajes más altos que el grupo de 15-16 años $(\mathrm{M}=1.25 ; \mathrm{DE}=.53)$.

\section{Diferencias por grado escolar}

Frecuencia de conflictos. Pudo observarse una cierta tendencia del promedio general a aumentar con el grado escolar siendo de $1.47(\mathrm{DE}=$ $.36), 1.51(\mathrm{DE}=.34)$ y $1.56(\mathrm{DE}=.32)$ para el Primero, Segundo y Tercer año respectivamente. Sin embargo, esta diferencia no resultó estadísticamente significativa.

En el análisis por temas de conflicto, se encontró diferencia estadísticamente significativa únicamente para los conflictos Por hacer ruido o estar hablando en el salón $\left(\mathrm{F}_{[2 / 168]}=7.240\right.$, $p<.01$ ), donde el análisis post hoc indicó que el grupo de Tercer año $(\mathrm{M}=2.06 ; \mathrm{DE}=.79)$ obtuvo puntajes más altos que los grupos de Primero $(\mathrm{M}=1.56 ; \mathrm{DE}=.74)$ y de Segundo $(\mathrm{M}=1.62$; $\mathrm{DE}=.70)$, de manera estadísticamente significativa $(p<.01$, en ambos casos).

Intensidad de conflictos. Pudo observarse una cierta tendencia del promedio general a aumen- tar con el grado escolar siendo de $1.47(\mathrm{DE}=$ $.37), 1.55(\mathrm{DE}=.42)$ y $1.62(\mathrm{DE}=.39)$ para el Primero, Segundo y Tercer año respectivamente. Sin embargo, esta diferencia no resultó estadísticamente significativa.

Una vez hecho el análisis por temas de conflicto, se encontraron diferencias estadísticamente significativas únicamente en dos temas de conflicto. En los conflictos Por burlas ( $\mathrm{F}_{[2 / 168]}$ $=3.676, p<.05)$ el análisis post hoc indicó que el grupo de Segundo año $(\mathrm{M}=2.09 ; \mathrm{DE}=.96)$ obtuvo puntajes más altos que el grupo de Primero $(\mathrm{M}=1.65 ; \mathrm{DE}=.79)$ con $p<.05$. Por su parte, en los conflictos Por hacer ruido o estar hablando en el salón $\left(\mathrm{F}_{[2 / 168]}=6.799, p<.01\right)$, el análisis post hoc indicó que el grupo de Tercer año $(\mathrm{M}=2.08 ; \mathrm{DE}=.82)$ obtuvo puntajes más altos que los grupos de Primero $(\mathrm{M}=1.53$; $\mathrm{DE}=.76)$ y de Segundo $(\mathrm{M}=1.64 ; \mathrm{DE}=.87)$, de manera estadísticamente significativa $(p<$ .01 y $p<.05$, respectivamente).

\section{Análisis correlacional}

Como puede observarse en la Tabla 3, los tres estilos de manejo de conflictos tuvieron correlaciones directas estadísticamente significativas tanto con Frecuencia como con Intensidad de conflictos; sin embargo, la fuerza de dichas correlaciones fue mayor para el estilo Centrado en Uno Mismo, seguido del estilo Centrado en la Otra Parte y, por último, del estilo Centrado en el Problema.

Tabla 3

Coeficientes de correlación Pearson entre estilos de manejo de conflictos y Frecuencia e Intensidad de conflictos.

\begin{tabular}{lccc}
\hline & $\begin{array}{c}\text { Centrado en } \\
\text { Uno Mismo }\end{array}$ & $\begin{array}{c}\text { Centrado en } \\
\text { la Otra parte }\end{array}$ & $\begin{array}{c}\text { Centrado en } \\
\text { el Problema }\end{array}$ \\
\hline Frecuencia de conflictos & $.381^{* * *}$ & $.228^{* *}$ & $.174^{*}$ \\
Intensidad de conflictos & $.347^{* * *}$ & $.221^{* *}$ & $.162^{*}$ \\
\hline
\end{tabular}

Nota. $\mathrm{N}=171 . * p<.05, * * p<.01, * * * p<.001$. 


\section{Discusión}

Como se señaló, la puntuación media total de Frecuencia e Intensidad de conflictos en ambos casos se ubicó en un lugar intermedio entre el punto 1 de la escala (Ningún conflicto) y el punto 2 (Algunos conflictos y Conflictos leves). Igualmente es de interés observar que todas las puntuaciones medias en todos los temas de conflicto se ubicaron en ese rango (entre el punto 1 y el 2 de la escala), tanto en Frecuencia como en Intensidad. Lo anterior indica que, en general, los participantes tendieron a reportar un nivel percibido bajo de incidencia de conflictos con sus compañeros. Este resultado coincide con los estudios de Luna-Bernal et al. (2015) y Luna-Bernal y Laca-Arocena (2011) realizados con adolescentes de secundaria y de bachillerato, respectivamente. Igualmente, en el estudio de Castro-Guerrero et al. (2011), los estudiantes tendieron a reconocer la existencia de conflictos en sus relaciones como normales, lo que parece indicar que, probablemente, los estudiantes tienden a interpretar los conflictos que se presentan en sus relaciones cotidianas como situaciones que no alteran gravemente su convivencia diaria.

Con relación a los temas de conflicto que obtuvieron las puntuaciones más elevadas, tanto en Frecuencia como en Intensidad, éstos comprendían los conflictos Por chismes, Porque algunos se creen más que los demás, Por falta de respeto o insultos y Por burlas. Este resultado coincide con el de Luna-Bernal et al. (2015) donde fueron precisamente estos mismos cuatro temas de conflicto los más frecuentes e intensos entre alumnos de secundaria. Asimismo, es congruente con los estudios de Delpino-Goicochea (2011) y Eresta-Plasín y Delpino-Goicochea (2009), donde los conflictos por burlas ocuparon el primer lugar entre los más frecuentes. En relación con esto, es interesante considerar que los cuatro temas de conflicto referidos pueden ubicarse dentro de los conflictos de relaciones
(Delpino-Goicochea, 2011; Eresta-Plasín \& Delpino-Goicochea, 2009; Moore, 1986/2010). En tales conflictos lo que está en juego es la naturaleza de la relación entre las partes. En dichos conflictos el elemento común sería que hay una situación de incompatibilidad percibida entre lo que uno espera de una relación y lo que la otra parte espera o hace.

Con relación a las diferencias de género, en el tema relativo a conflictos Por dinero, los hombres obtuvieron puntajes más altos que las mujeres de manera estadísticamente significativa, tanto en frecuencia como en intensidad de conflictos. Este resultado concuerda, por un lado, con el estudio citado por Denegri-Coria (2004), de acuerdo con el cual "en los sujetos de entre 15 a 19 años se apreció la coexistencia de fuentes formales de endeudamiento como el comercio y las tarjetas de crédito, con fuentes informales como los amigos y las familias" ( $p$. 34). Esto podría indicar la existencia de préstamos y relaciones dinerarias entre los adolescentes. Por otro lado, el hallazgo del presente estudio también concuerda con algunos trabajos que se han realizado sobre actitudes hacia el dinero en jóvenes y adolescentes donde se ha encontrado que, en general, los varones tienden a interpretar la posesión de dinero como un símbolo de poder, éxito y felicidad, mientras que las mujeres piensan en él en términos más funcionales (Santa-María-Ledesma \& Gómez-Lafuente, 2005). Así, la probable existencia de relaciones crediticias entre los adolescentes, unida a una mayor importancia como símbolo de poder y estatus dada por los varones al dinero, podría explicar por qué ellos tienden a presentar mayor frecuencia e intensidad de conflictos con respecto a este tema.

Por otra parte, en el tema relativo a conflictos Por hipocresía, las mujeres obtuvieron puntajes más altos que los hombres de manera estadísticamente significativa, tanto en Frecuencia como en Intensidad de conflictos. Este resul- 
tado es congruente con la literatura sobre agresión en la que se ha reportado consistentemente una mayor inclinación de las mujeres hacia la agresión relacional o indirecta. De acuerdo con Arnett (2007/2008), "las muchachas recurren a la agresión en las relaciones porque su papel de género prohíbe las expresiones más directas de desacuerdo y conflictos" (p. 253). De acuerdo con Björkqvist (2007), "las mujeres no solamente exceden a los hombres en agresión indirecta, sino también ellas son mejores que ellos en la resolución pacífica de los conflictos interpersonales" (p. 80). Según este último autor, ello se debe a que ambos tipos de conducta de conflicto requieren un grado relativamente alto de inteligencia social, habilidad que las mujeres desarrollan más rápido que los hombres (aunque la diferencia declina con la edad). Si, de acuerdo con Björkqvist (2007), las mujeres adolescentes son socialmente más competentes que los varones, tal competencia puede ser utilizada para ambos propósitos, agresivos o pacíficos. Este resultado puede contribuir a la explicación acerca de por qué las mujeres adolescentes tienden a aventajar a los varones tanto en agresión indirecta como en resolución pacífica de conflictos, y podría indicar que, habiendo una mayor incidencia de agresión relacional, es probable que se origine mayor incidencia de conflictos con respecto a esas conductas.

En el tema relativo a conflictos Por la división de tareas en equipo, las mujeres obtuvieron puntajes más altos que los hombres de manera estadísticamente significativa, tanto en Frecuencia como en Intensidad de conflictos. Una posible explicación de este resultado podría estar relacionada con el significado que los estudiantes hombres y mujeres atribuyen a su experiencia de estudiar el bachillerato. Con relación a esto, Guerra-Ramírez (2000) realizó un estudio en el cual entrevistó a estudiantes de bachillerato en el que encontró que las mujeres tienen mayores expectativas de proseguir estudios superiores, como una estrategia para enfrentar desigualdades de género a futuro. De acuerdo con el autor, el bachillerato es percibido por las mujeres como una alternativa a esa situación de desigualdad, mientras que los hombres lo ven como "algo que debe hacerse". Así, según Guerra-Ramírez (2000), mientras que entre los varones el bachillerato tiende a ser percibido como algo no-elegido en mayor grado que en las mujeres, ellas probablemente tienden a percibirlo más como una elección personal. De acuerdo con lo anterior, sería entonces probable que las mujeres tendieran, por lo general, a sentir una mayor implicación y responsabilidad personal en los resultados de los estudios en el bachillerato, lo cual podría verse reflejado en aquellas situaciones en las que se les pide hacer trabajos en equipo.

Con relación a las diferencias por edad, el grupo de 17-19 años obtuvo puntajes más altos que el grupo de 15-16 años de manera estadísticamente significativa en los conflictos Por la manera de ser de uno u otro. Este resultado podría estar relacionado con el desarrollo de la identidad personal, como un proceso característico de la adolescencia (Arnett, 2007/2008). A este respecto es probable que los adolescentes de mayor edad hayan logrado establecer de manera más definida una serie de características personales como parte de su identidad. De este modo, es probable que también resulten ser más proclives a discutir sobre este tema cuando interactúan con compañeros que han asumido como constitutivas de su propia identidad características diferentes, o incluso contrarias.

Con relación a las diferencias por grado, el grupo de Segundo año obtuvo puntajes más altos que el grupo de Primero de manera estadísticamente significativa en los conflictos Por burlas. De acuerdo con Arnett (2007/2008), los adolescentes aprecian y usan el sarcasmo más que los niños y preadolescentes, debido a la mayor capacidad para el pensamiento complejo. Además, el uso del sarcasmo y el ridículo hacia las personas externas al grupo de amigos tam- 
bién fortalece la identidad del grupo de amigos. Siguiendo este planteamiento, sería de esperar que el ejercicio del sarcasmo, el ridículo y las burlas fuera más sistemático en un contexto donde los grupos de amistad estén mayormente formados, respecto de otro contexto donde dichos grupos no tienen el mismo grado de consolidación. Así, si dentro de un aula de clase los grupos de amigos en los primeros grados están menos definidos que en los grados intermedios, sería de esperar que en estos últimos se presentara de manera más sistemática el uso del sarcasmo, el ridículo y las burlas y, por lo tanto, una mayor incidencia de conflictos relativos a estos temas.

Por otra parte, en los conflictos Por hacer ruido o estar hablando en el salón el grupo de 17-19 años obtuvo puntajes más altos que el grupo de 15-16 y asimismo, el grupo de Tercer año obtuvo puntajes más altos que los grupos de Primero y de Segundo año, de manera estadísticamente significativa, tanto en Frecuencia como en Intensidad de conflictos. De acuerdo con Delpino-Goicochea (2011), "las conductas perturbadoras del orden y de la tranquilidad en el ambiente escolar afectan las metas de aquellos estudiantes motivados por atender a algunos temas o que manifiestan interés por determinadas actividades desarrolladas en las clases" (p. 191). De esta manera, es probable que entre los alumnos de mayor edad y grado escolar sea posible encontrar un sector que esté positivamente motivado hacia el aprendizaje y la realización de las actividades escolares, sobre todo tomando en cuenta sus expectativas de concluir el bachillerato para proseguir estudios superiores. Si junto a este sector de estudiantes se encontrara, dentro del mismo grupo, otro sector que no esté motivado en ese sentido, se presentaría una incompatibilidad de intereses de uno y otro sector originándose conflictos relativos a estos temas.

Pasando a los resultados del análisis correlacional, en el presente estudio se encontró: a) que los tres estilos de manejo de conflictos presentaban correlaciones directas estadísticamente significativas tanto con Frecuencia como con Intensidad de conflictos; b) que la fuerza de dichas correlaciones era mayor para el estilo Centrado en Uno Mismo, seguido del estilo Centrado en la Otra Parte y, finalmente, del estilo Centrado en el Problema.

En primer lugar, es evidente que una mayor incidencia de conflictos implica mayores oportunidades para hacer uso de todos los estilos de manejo de conflictos. Así, un mayor uso de todos los estilos podría ser solamente el efecto de la necesidad que se presenta a los adolescentes de afrontar una mayor cantidad de conflictos.

En segundo lugar, la diferencia en la fuerza de las correlaciones podría indicar que, a medida que aumenta la magnitud de conflictos, los adolescentes tienden a emplear unos estilos con mayor frecuencia que otros. Los resultados parecen indicar que, a medida que aumenta la magnitud de los conflictos, los adolescentes tienden a dar mayor preferencia al estilo Centrado en Uno Mismo, seguido del Centrado en la Otra Parte y, en tercer término, del Centrado en el Problema. Ello es congruente con el fenómeno, reiteradamente reportado en la literatura sobre análisis de conflictos, de que los conflictos que son manejados a través de estilos competitivos tienden muchas veces a escalar (Rubin et al., 1994).

Una limitación que es importante anotar con relación a este último hallazgo es el hecho, ya referido, de que los adolescentes reportaron niveles bajos de Frecuencia e Intensidad de conflictos en el presente estudio. Debido a ello, en el presente caso, las correlaciones entre ambos grupos de variables (frecuencia e intensidad de conflictos y estilos de manejo de conflictos) corresponden al rango de magnitud de conflictos de bajo a moderado, pero no indican qué sucedería con dichas correlaciones en grupos donde la magnitud de conflictos alcanzara niveles moderados y altos. Por lo anterior, sería 
recomendable que en la investigación futura se contemple la posibilidad de evaluar las relaciones entre la magnitud de conflictos y sus estilos de gestión en contextos de alta incidencia, con el fin de identificar si se mantiene una relación directa entre ambos grupos de variables.

\section{Referencias}

Arnett, J. J. (2008). Adolescencia y adultez emergente. Un enfoque cultural ( $3^{\circ}$ ed.; Trad. M. E. Ortiz). México: Pearson Educación. (Trabajo original publicado en 2007).

Bascón, M., Saavedra, J., \& Arias, S. (2013). Conflictos y violencia de género en la adolescencia. Análisis de estrategias discursivas y recursos para la coeducación. Profesorado. Revista de Curriculum y Formación del Profesorado, 17(1), 289-307. Recuperado de http://www.ugr.es/ recfpro

Björkqvist, K. (2007). Empathy, social intelligence and aggression in adolescent boys and girls. En T. F. D. Farrow \& P. W. R. Woodruff (Eds.), Empathy in mental illness (pp. 76-88). Cambridge: Cambridge University Press.

Castro-Guerrero, P., López-Jiménez, E., \& Meza-Altamar, Y. E. (2011). Diagnóstico de los conflictos estudiantiles y su manejo, para el diseño de unidades conceptuales en una cartilla pedagógica. Escenarios, 9(2), 58-69. Recuperado de https://dialnet. unirioja.es/servlet/articulo? codigo $=4495535$

Coolican, H. (2005). Métodos de investigación y estadistica en psicología ( $3^{\circ}$ ed.; Trads. G. Padilla-Sierra, S. M. Olivares-Bari, \& J. L. Núñez-Herrejón). México: Manual Moderno. (Trabajo original publicado en 2004).

Delpino-Goicochea, M. A. (2011). Inmigración y educación. El conflicto en la escuela española y sus percepciones. (Tesis doctoral). Recuperado de http:// hdl.handle.net/10366/115582

Denegri-Coria, M. (2004). Introducción a la Psicología Económica. Bogotá: Psicom Editores.

Eresta-Plasín, M. J., \& Delpino-Goicochea, M. A. (2009). Conflictos en la adolescencia: Los protagonis- tas toman la palabra. Madrid: Liga Española de la Educación de Utilidad Pública y Gobierno de España, Ministerio de Sanidad, Política Social e Igualdad. Recuperado de http://www.ligaeducacion.org/documentos/investigaciones/conflictos-en-la-adolescencia.pdf

Filley, A. C. (1985). Solución de conflictos interpersonales (Trad. C. Villegas). México: Trillas. (Trabajo original publicado en 1975).

Fry, D. (2006). The human potential for peace. An anthropological challenge to assumptions about war and violence. New York: Oxford University Press.

Galtung, J. (2003). Paz por medios pacíficos. Paz y conflicto, desarrollo y civilización (Trad. T. Toda). Bilbao: Bakeaz y Gernika Gogoratuz.

Guerra-Ramírez, M. I. (2000). ¿Qué significa estudiar el bachillerato? La perspectiva de los jóvenes en diferentes contextos socioculturales. Revista Mexicana de Investigación Educativa, 5(10), 243-272. Recuperado de http://www.comie.org.mx/v1/revista

IBM (2012). SPSS Statistics 21.0.0. [software de cómputo]. Recuperado de https://www-01.ibm.com/ software/mx/analytics/spss/products/statistics

Laca, F. A., Mejía, J. C., \& Mayoral, E. G. (2011). Conflict communication, decision-making, and individualism in Mexican and Spanish university students. Psychology Journal, 8(1), 121-135.

Laursen, B., \& Collins, W. A. (1994). Interpersonal conflict during adolescence. Psychological Bulletin, 115(2), 197-209. doi: 10.1037/00332909.115.2.197

Laursen, B., \& Koplas, A. (1995). What's important about important conflicts? Adolescents' perceptions of daily disagreements. Merrill-Palmer Quarterly, 41(4), 536-553. Recuperado de http://www.jstor. org/stable/23087940

Luna, A. C. A., \& Laca, F. A. V. (2014). Estilos de mensajes en el manejo de conflictos en adolescentes y jóvenes mexicanos. Boletín de Psicología, 110, 37-51. Recuperado de http://www.uv.es/seoane/ boletin/boletin.html

Luna-Bernal, A. C. A. (2014). Efecto de la comunicación parento-filial sobre los estilos personales de manejo de conflictos en adolescentes bachilleres. Uari- 
cha, Revista de Psicología, 11(24), 118-133. Recuperado de http://www.revistauaricha.umich.mx

Luna-Bernal, A. C. A., \& Cruz-Abundis, C. (2014). Frecuencia e intensidad de conflictos con los padres en adolescentes bachilleres. Revista Alternativas en Psicología, 18(30), 8-21. Recuperado de http:// alternativas.me

Luna-Bernal, A. C. A., \& De Gante-Casas, A. (2017). Empatía y gestión de conflictos en estudiantes de secundaria y bachillerato. Revista de Educación y Desarrollo, 40, 27-37. Recuperado de http://www. cucs.udg.mx/revistas/edu_desarrollo

Luna-Bernal, A. C. A., De Gante-Casas, A., \& Gómez-Pérez, M. A. (2015). Violencia escolar y afrontamiento de conflictos en adolescentes de educación secundaria. Guadalajara, Jalisco (México): Consejo Estatal de Ciencia y Tecnología del Estado de Jalisco (COECYTJAL) y Universidad de Guadalajara.

Luna-Bernal, A. C. A., \& Laca-Arocena, F. A.V. (2011). Frecuencia e intensidad de conflictos entre adolescentes bachilleres. Documento de trabajo inédito. Departamento de Filosofía, Universidad de Guadalajara, y Facultad de Psicología, Universidad de Colima. Guadalajara y Colima, México.

Luna-Bernal, A. C. A., Laca-Arocena, F. V., \& Cruz-Abundis, M. C. (2013). Conflictos con los padres y satisfacción con la vida de familia en adolescentes de secundaria. Revista Electrónica de Psicología Iztacala, 16(3), 864-887. Recuperado de http://www. revistas.unam.mx/index.php/repi

Martínez-Guzmán, V. (2009). Filosofía para hacer las paces $\left(2^{\circ}\right.$ ed.). Barcelona: Icaria Editorial.

Mejía-Ceballos, J. C., \& Laca-Arocena, F. A. (2006). Estilos de comunicación en el conflicto y confianza en las propias decisiones. Enseñanza e Investigación en Psicología, 11(2), 347-358. Recuperado de http://www.redalyc.org/articulo.oa?id=29211210

Moore, C. W. (2010). El proceso de mediación. Métodos prácticos para la resolución de conflictos (Trad. A. Leal). Buenos Aires: Granica. (Trabajo original publicado en 1986).

Pantoja-Vallejo, A. (2005). La gestión de conflictos en el aula. Factores determinantes y propuestas de intervención. En A. Rivera-Otero \& M. Pérez-Solís
(Eds.), Orientación escolar en centros educativos (pp. 319-357). Jaén, España: Ministerio de Educación y Ciencia, Secretaría General Técnica. Recuperado de https://sede.educacion.gob.es/publiventa/detalle.action? $\operatorname{cod}=11828$

Paris-Albert, S. (2009). Filosofia de los conflictos. Una teoría para su transformación pacífica. Barcelona: Icaria Editorial.

Parra-Jiménez, A., \& Oliva-Delgado, A. (2002). Comunicación y conflicto familiar durante la adolescencia. Anales de Psicología, 18(2), 215-231. Recuperado de http://revistas.um.es/analesps

Pérez-Ramos, M., \& Pineda-Sánchez, E. R. (2013). Relación entre el sexo y la frecuencia e intensidad del conflicto entre padres y adolescentes. Revista de Psicología Social y Personalidad, 29(1), 49-60.

Ritchey, F. J. (2008). Estadística para las ciencias sociales ( $2^{\circ}$ ed.; Trads. J. H. Romo-Muñoz, J. Yescas-Milanes, J. León-Cárdenas, \& J. A. Velázquez-Arellano). México: McGraw-Hill Interamericana. (Trabajo original publicado en 2008).

Ross, R., \& DeWine, S. (1988). Assessing the Ross-DeWine Conflict Management Message Style (CMMS). Management Communication Quarterly, 1, 389413. doi: 10.1177/0893318988001003007

Rubin, J. Z., Pruitt, D. G., \& Kim, S. H. (1994). Social conflict: Escalation, stalemate and settlement $\left(2^{\circ}\right.$ ed.). New York: McGraw-Hill Inc.

Santa-María-Ledesma, C., \& Gómez-Lafuente, J. (2005). Actitudes hacia el dinero en jóvenes de 18 a 23 años. Ajayu, 3(1), 86-107. Recuperado de http:// www.ucb.edu.bo/publicaciones/ajayu/revista.html 